\title{
Dimensionamento de sistemas híbridos e autônomos para iluminação de ruas em áreas isoladas
}

Dimensioning of hybrid and autonomous systems for street lighting in isolated areas

\section{Erikson Carlos Ramos ${ }^{\mathbf{1}}$ (Dorcid.org/0000-0002-8960-3072}

José Bione de Melo Filho' ${ }^{1}$ orcid.org/0000-0002-9283-3362

${ }^{1}$ Escola Politécnica de Pernambuco, Universidade de Pernambuco, Recife, Brasil,

E-mail do autor principal: Erikson Carlos Ramos erikson.carlos@hotmail.com

\section{RESUMO}

Este trabalho apresenta o dimensionamento básico de um sistema autônomo de iluminação pública baseado em fontes renováveis de energia solar e eólica, como fontes primárias, baterias, como fonte secundária, e diodos emissores de luz (LEDs), como fonte luminosa. Este sistema é apresentado como uma alternativa de iluminação para locais remotos, tais como rodovias, localidades isoladas e pontos turísticos. Para tanto, são apresentadas características básicas dos componentes que compõe tal sistema como: luminária, painéis solares, aerogerador, controlador de carga e banco de baterias. A metodologia proposta consiste em pesquisa bibliográfica sobre sistemas voltaicos, eólicos, autônomos e de iluminação.

PALAVRAS-CHAVE: Baterias; Iluminação Pública; Energia Solar; Energia Eólica;

\section{ABSTRACT}

This paper presents an autonomous system of public lighting based on renewable sources of solar and wind energy, as primary sources, secondary sources, such as batteries and lightemitting diodes (LED), as light source. This system is presented as a lighting alternative for remote locations, such as highways, clovers, isolated locations and sights. For this purpose, are presented basic characteristics of the components that make up such a system as light, solares panels, wind generator, charge controller and battery bank. The proposed methodology consists of bibliographic research on wind systems, photovoltaic, autonomous and lighting.

KEY-WORDS: Batteries, Street Lighting, Solar Energy and Wind Energy. 


\section{INTRODUÇÃO}

Nos últimos anos o Brasil tem passado por um aumento no custo de energia elétrica devido à diminuição dos níveis dos reservatórios, o que tem obrigado o acionamento das usinas termelétricas, que possuem um custo de geração mais elevado que as usinas hidrelétricas. Esseaumento no custo da energia elétricavem sendo sinalizado através do controle de bandeiras tarifárias, criado a partir do ano de 2015 e inserido na conta de energia enviada pelas concessionárias [1].

Nesse cenário de aumento de custo de energia elétrica, surge a necessidade de se criarem alternativas para o suprimento do sistema elétrico nacional, ou seja, não se limitar a fontes hidrelétricas.

Considerando os aspectos mencionados e visando uma possível solução para iluminação de arruamentos em regiões isoladas, trechos de rodovias, indústrias com grandes extensões de área, entre outras aplicações similares, este trabalho propõe apresentar de forma simplificada e objetiva, como uma alternativa ao abastecimento de energia nas áreas citadas, um sistema híbrido (energia solar e eólica) e autônomo (baterias).

Tendo em vista o contexto apresentado, é importante ressaltar que sistemas para a geração de energia elétrica a partir de fontes renováveis de energia, apresentam cada vez mais contribuições energéticas, com o mínimo impacto ambiental, sendo bastante importante aprofundarse nos estudos destas tecnologias [2].

Em relação a tecnologia eólica-fotovoltaica, é importante frisar que a radiação solar e o e a energia dos ventos podem se mostrar complementares para alguns locais. Assim, para determinadas aplicações esses sistemas podem apresentar algumas vantagens em relação aos que utilizam apenas uma dessas tecnologias, pois a confiabilidade do sistema é reforçada [2].

Sendo assim, para locais isolados o uso de sistemas híbrido eólico-fotovoltaico pode ser ineficiente se considerarmos que determinados dias não chove e tão pouco venta. Então, surge a necessidade de uma complementação, que pode ser um sistema híbrido eólico-fotovoltaico e autônomo com a utilização de baterias. Essa arquitetura é complementada com a presença de um controlador de carga.
Em síntese, a Figura 1 mostra a arquitetura do sistema híbrido eólico-fotovoltaico e autônomo, escopo deste trabalho.

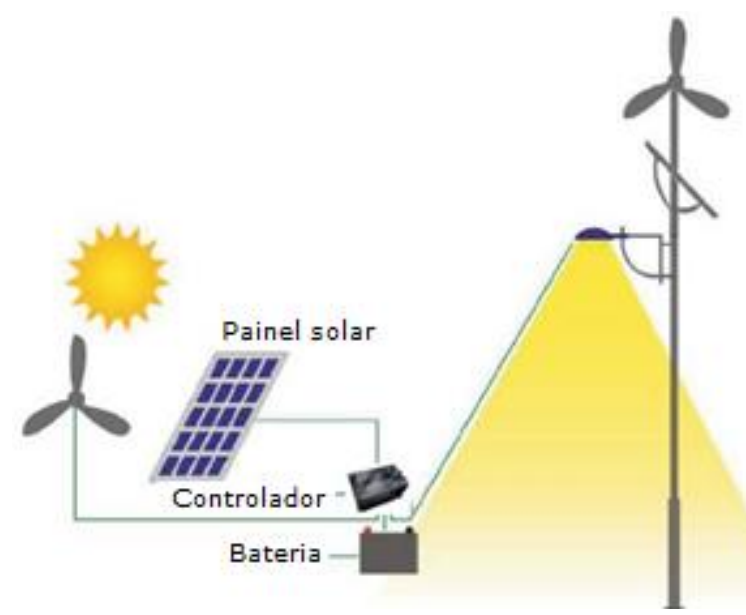

Figura 1:Sistema híbrido eólico-fotovoltaico autônomo. Fonte:Imagem adaptada de [3].

Nesse sentido, vale ressaltar que o objetivo deste artigo é descrever os passos a serem seguidos e os detalhes a serem observados para o desenvolvimento de um sistema híbrido eólicofotovoltaico e autônomo. No entanto, diversos outros estudos e projetos devem ser feitos para a escolha de um sistema de iluminação para áreas isoladas, principalmente, um estudo de viabilidade. Atualmente, os preços dos equipamentos têm sofrido alterações significativas e podem inviabilizar uma configuração como a apresentada, dessa forma, há uma importância de um estudo de viabilidade para um caso específico, com as características do ambiente e demais variáveis envolvidas.

Por fim, salienta-se que o estudo, do ponto de vista do objetivo, foi desenvolvido de forma descritiva. Em relação ao ponto de vista da natureza, na forma de aplicação prática e, quanto aos procedimentos empregados no estudo, utilizou-se da pesquisa bibliográfica. Por último, em relação a abordagem do problema, a pesquisa teve uma abordagemqualitativa.

Dito isso, a seguir são detalhados os componentes do sistema apresentado na Figura 1.

\section{Componentes de sistemas híbridos eólicos-fotovoltaico e autônomos}

Os componentes de um sistema híbrido eólicofotovoltaico e autônomo que necessitam ser dimensionados e detalhados são os seguintes: sistema de iluminação, sistema eólico 
(aerogerador), painéis fotovoltaicos, sistema de armazenamento de energia (baterias) e o controlador de carga. Prosseguindo-se, a seguir, são apresentadas as características doscomponentes do sistema em análise.

\subsection{Sistema de iluminação}

O objetivo deste tópico é de esclarecer como é feita a escolha da luminária a ser utilizada no sistema. Ressalta-se que, objetivo do estudo em questão não é detalhar os cálculos necessários para a determinação da luminária do sistema em análise, por isso, serão adotadas premissas para se definir um equipamento que possa ser utilizado como referência para o dimensionamento dos demais componentes da arquitetura híbrida apresentada. Para um caso concreto deve-se fazer estudos luminotécnicos e, também, de viabilidade, ponderando o custo-benefício de cada luminária disponível no mercado. Um software bastante utilizado em estudos luminotécnico é o DiaLux da Dial.

Considerando o contexto apresentado e as recentes tecnologias aplicadas a sistemas de iluminação, apesar de ter sido inventado em 1961, os Light Emitting Diodes (LED ${ }^{1} \mathrm{~s}$ ), diodos emissores de luz, somente, chegaram ao ramo de iluminação em 1999. Atualmente, várias cidades já iniciaram sua adoção na iluminação de vias públicas em contrapartida as luminárias de vapor de sódio. Sendo assim, no estudo proposto será adotada uma luminária com lâmpada LED. Antes, porém, será necessário o entendimento da iluminação de vias públicas, na concepção das concessionárias de energia elétrica [4].

No estado de Pernambuco a concessionária responsável por dar as diretrizes e padrões no serviço de iluminação pública é a Companhia Energética de Pernambuco (CELPE), empresa pertencente ao Grupo Neoenergia. Para essa concessionária a iluminação pública nada mais é que um serviço público que tem por objetivo exclusivo iluminar os logradouros públicos, de forma periódica, contínua ou eventual [5].

Em relação aos padrões (altura de instalação da luminária, potência da lâmpada, espaçamento

\footnotetext{
${ }^{1}$ LED é um componente eletrônico dotado de cristal semicondutor de silício ou germânio que produz fotoluminescência. O nome dado é uma sigla de Light Emitting Diode ou Diodo emissor de luz [4].
}

entre luminárias e outros) adotados pela concessionária, há detalhes que precisam ser considerados, como: classe de iluminação da via, iluminação média mínima exigida pela via, vão médio a ser considerado na instalação das luminárias e altura de montagem mínimanecessária.

Nesse sentido, considerar-se-á, inicialmente, os padrões da CELPE para uma predefinição da carga.Todavia, existem iluminação de vias, como de condomínios fechados, de empresas e outras, em áreas isoladas, que não necessariamente vão seguir o padrão da concessionária de energia local. É possível que, em alguns casos, seja necessário, apenas, utilizar as normas da ABNT Associação Brasileira de Normas Técnicas. Assim, a seguir são adotadas algumas premissas para a definição da carga que será utilizada como referência na arquitetura em questão.

De acordo com os padrões da CELPE [5], existem 3 tipos de lâmpadas utilizadas na iluminação pública: vapor de sódio (VS) de 70W, $150 \mathrm{~W}$ e $250 \mathrm{~W}$.

O que definirá qual a potência a ser utilizada é a classificação do tipo de via e fluxo de veículos. O Quadro 1 define a classificação quanto ao volume de tráfego de veículos motorizados em vias públicas [6].

Quadro 1: Classificação das vias quanto ao volume de tráfego.

\begin{tabular}{|c|c|}
\hline Classificação & $\begin{array}{l}\text { Volume de tráfego noturno }{ }^{a} \text { de } \\
\text { veículos por hora, em ambos os } \\
\text { sentidos }^{b} \text {, em pista única }\end{array}$ \\
\hline Leve (L) & 150 a 500 \\
\hline Médio (M) & 501 a 1200 \\
\hline Intenso (I) & Acima de 1200 \\
\hline \multicolumn{2}{|c|}{$\begin{array}{l}\text { Valor máximo das médias horárias obtidas nos períodos } \\
\text { compreendidos entre } 18 \text { h e } 21 \mathrm{~h} \text {. } \\
\text { Valores para velocidades regulamentadas por lei. } \\
\text { NOTA: Para vias com tráfego menor do que } 150 \text { veículos } \\
\text { por hora, consideram-se as exigências mínimas do grupo } \\
\text { leve (L) }\end{array}$} \\
\hline
\end{tabular}

Fonte: [6].

Quadro 2 define a classe de iluminação para cada tipo de via para tráfego de veículos [6]. 
Quadro 2: Classes de iluminação para cada tipo de via. (Continua)

\begin{tabular}{|l|l|}
\hline Descrição da via & Classe de iluminação \\
\hline $\begin{array}{l}\text { Vias de trânsito rápido; vias de alta velocidade de } \\
\text { tráfego, com separação de pistas, sem } \\
\text { cruzamentos em nível e com controle de acesso; } \\
\text { vias de trânsito rápido em geral; Autoestradas: }\end{array}$ \\
\hline Volume de tráfego intens & V1 \\
\hline Volume de tráfego médid & V2 \\
\hline $\begin{array}{l}\text { Vias arteriais; vias de alta velocidade de tráfego } \\
\text { com separação de pistas; vias de mão dupla, com } \\
\text { cruzamentos e travessias de pedestres eventuais } \\
\text { em pontos bem definidos; vias rurais de mão dupla } \\
\text { com separação por canteiro ou obstáculo: }\end{array}$ \\
\hline $\begin{array}{l}\text { Volume de tráfego } \\
\text { intenso }\end{array}$ & V1 \\
\hline Volume de tráfego médi & V2 \\
\hline $\begin{array}{l}\text { Vias coletoras; vias de tráfego importante; vias } \\
\text { radiais e urbanas de interligação entre bairros, com } \\
\text { tráfego de pedestres elevado: }\end{array}$ \\
\hline $\begin{array}{l}\text { Volume de tráfego } \\
\text { intenso }\end{array}$ & V2 \\
\hline Volume de tráfego médii & V3 \\
\hline Volume de tráfego leve & V4 \\
\hline de acesso residencial: \\
\hline Volume de tráfego médii & V4 \\
\hline Volume de tráfego leve & V5 \\
\hline
\end{tabular}

Fonte: [6].

Já oQuadro 3 define a iluminância média mínima para cada classe de iluminação de via [6].

Quadro 3: Requisitos mínimos de iluminância média mínima para cada classe de iluminação.

\begin{tabular}{|l|l|}
\hline Classificação & $\begin{array}{l}\text { Iluminância média mínima } \\
\text { Eméd.min.lux }\end{array}$ \\
\hline V1 & 30 \\
\hline V2 & 20 \\
\hline V3 & 15 \\
\hline V4 & 10 \\
\hline V5 & 5 \\
\hline
\end{tabular}

Fonte: [6].

Além dos Quadros 1, 2 e 3 ainda se tem um quadro que mostra a configuração da grade de referência (vão médio, altura de montagem, espaçamento e etc.) de acordo com a classe de iluminação da via, que é o Quadro 4 [6].

Quadro 4: Configuração da grade de referência de acordo com a classe de iluminação da via.

\begin{tabular}{|l|l|l|l|l|l|}
\hline $\begin{array}{l}\text { Classificação de } \\
\text { iluminação da via }\end{array}$ & $\mathbf{V 1}$ & $\mathbf{V 2}$ & $\mathbf{V 3}$ & $\mathbf{V 4}$ & $\mathbf{V 5}$ \\
\hline Vão médio (m) & 40 & 35 & 35 & 35 & 35 \\
\hline Altura de montagem (m) & 12 & 9 & 8 & 8 & 7 \\
\hline $\begin{array}{l}\text { Número de faixas de } \\
\text { trânsito da via }\end{array}$ & 4 & 4 & 3 & 3 & 3 \\
\hline $\begin{array}{l}\text { Largura por faixa da via } \\
\text { (m) }\end{array}$ & 3 & 2,7 & 3 & 3 & 2,7 \\
\hline $\begin{array}{l}\text { Largura total da via/calha } \\
\text { (m) }\end{array}$ & 9 & 8,1 & 9 & 9 & 8,1 \\
\hline Avanço ${ }^{a}(\mathrm{~m})$ & 3 & 2, & 1, & $\begin{array}{l}1, \\
5\end{array}$ & $\begin{array}{l}1, \\
5\end{array}$ \\
\hline
\end{tabular}

a $\mathrm{O}$ avanço corresponde à distância entre o início da grade sob a luminária (do meio-fio) até a extremidade do braço onde é montada a luminária.

Fonte: [6].

Em resumo, para projetos de iluminação pública, os seguintes parâmetros principais devem ser levados em consideração: classe de iluminação da via (V1, V2, V3, V4 e V5), iluminação média mínima ( $5,10,15,20$ e 30lux), vão médio (35 à $40 \mathrm{~m}$ ) e altura de montagem (7 à $12 \mathrm{~m}$ ). Com todos esses dados pode-se efetuar simulações com softwares específicos, como o DiaLux, e chegar nas potências adequadas das lâmpadas, bem como nos valores mais precisos de altura e espaçamento. Como a CELPE utiliza valores padronizados para as lâmpadas, e considerando a utilização de lâmpadas LED, pois estas apresentam um tempo de vida útil superior a $50.000 \mathrm{~h}$, pode-se utilizar o Quadro 5 [7]como referência para encontrar a lâmpada equivalente. Vale ressaltar que esse quadro apresenta uma aproximação simplificada e, para valores mais precisos, deve-se recorrer a simulações detalhadas e cálculos específicos, algo que não é o objetivo deste trabalho. 
Quadro 5: Equivalência entre lâmpada LED e lâmpadas convencionais.

\begin{tabular}{|l|l|}
\hline $\begin{array}{l}\text { Lâmpada } \\
\text { convencional } \\
\text { (Vapor de sódio) }\end{array}$ & Lâmpada LED Equivalente \\
\hline $150 \mathrm{~W}$ & $80 \mathrm{~W}$ \\
\hline $250 \mathrm{~W}$ & $130 \mathrm{~W}$ \\
\hline $400 \mathrm{~W}$ & $150 \mathrm{~W}$ \\
\hline
\end{tabular}

Fonte: [7].

Estima-se que o consumo mensal (30 dias) de uma lâmpada LED seja:

- Com uma potência de 150W, operando durante $12 \mathrm{~h} / \mathrm{dia}$, seja igual a: Consumo $=$ 30 dias $\times 12$ horas $/$ dia $\times 150 \mathrm{~W}=$ $54,0 \mathrm{kWh}$;

- Com uma potência de 130W, operando durante $12 \mathrm{~h} /$ dia, seja igual a: Consumo $=$ 30 dias $\times 12$ horas/dia $\times 130 \mathrm{~W}=$ 46,8kWh;

- Com uma potência de 80W, operando durante $12 \mathrm{~h} /$ dia, seja igual a: Consumo $=$ 30 dias $\times 12$ horas/dia $\times 80 \mathrm{~W}=28,8 \mathrm{kWh}$;

Neste caso, o sistema híbrido deverá ser capaz de suprir esses consumos mensais. Com intuito de simular, de forma básica, um sistema híbrido e autônomo será adotada uma luminária LED de $130 \mathrm{~W}$ (equivalente a uma de $250 \mathrm{~W}$ convencional), cujo consumo mensal é de $46,8 \mathrm{kWh}$ (Elâmpada). No entanto, poderia ter sido escolhida qualquer uma das opções para fins de simulação.

Por fim, com a escolha da luminária, Figura 2 [8], tem-se a potência que será utilizada no dimensionamento dos demais componentes da arquitetura em questão, conforme detalhado a seguir.

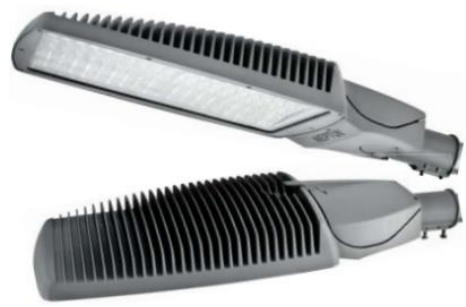

Figura 2: Luminária LED 130W/24V- Modelo LED-45130-24VDC da NEPTUN LIGHT.

Fonte: [8].

\subsection{Sistema eólico (Aerogerador)}

Para se especificar um aerogerador deve-se saber as condições do vento do local em que o equipamento será instalado. A partir disso a potência gerada por uma turbina é calculada através dos seguintes parâmetros: massa específica do ar, área de intercepção do rotor, velocidade do vento e eficiência do conjunto gerador/transmissão [9].

$$
\mathrm{P}=\frac{1}{2} \rho_{x} A r_{*} V^{3} C
$$

Onde:

$P=$ Potência em Watts;

$\rho=$ Densidade do ar em $\mathrm{kg} / \mathrm{m}^{3}$;

$\mathrm{Ar}=$ Área de varredura do rotor em $\mathrm{m}^{2}\left(\pi \cdot \mathrm{d}^{2} / 4-\right.$ $\mathrm{d}=$ diâmetro do rotor);

$\mathrm{V}=$ velocidade do vento em $\mathrm{m} / \mathrm{s}$;

$\mathrm{Cp}=$ coeficiente aerodinâmico de potência do motor (Máx. 0,45. Teórico 0,593); e

$\eta$ = eficiência do conjunto gerador/transmissões mecânicas e elétricas (0,93-0,98).

Considerando a finalidade do aerogerador que deve ser utilizado e adotando algumas premissas é possível calcular a potência do aerogerador eólico. Sendo assim, as seguintes premissas serão adotadas:

- $\rho=1,125 \mathrm{~kg} / \mathrm{m}^{3}$ (a densidade do ar é determinada pela sua temperatura $e$ pressão, e estas variáveis dependem da altitude em relação ao nível do mar, e este valor fica entre 1 e $1,25 \mathrm{~kg} / \mathrm{m}^{3}$, devido a isso será adotado o valor intermediário) [10];

- Ar $=1,13 \mathrm{~m}^{2}$ (aerogerador com, aproximadamente, $1,2 \mathrm{~m}$ de diâmetro tamanho razoável para este tipo de aplicação);

- V = variável (ver Figura5);

- $\mathrm{Cp}=0,45$;

$-\eta=0,93$.

No Brasil a velocidade média do vento é menor que $6 \mathrm{~m} / \mathrm{s}$. Neste caso, deve-se escolher uma turbina que opere nessa faixa de velocidade de vento. Para este estudo será adotada a turbina Air Breeze mostrada na Figura 3 [11], pois ela é capaz de gerar $38 \mathrm{kWh}$ por mês com uma velocidade média de vento de $5,4 \mathrm{~m} / \mathrm{s}$, ou seja, um valor muito próximo da velocidade média do vento no Brasil. 


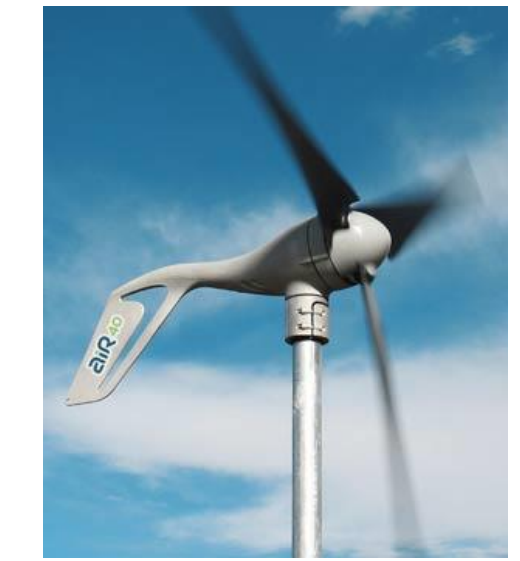

Figura 3: Aerogerador Air Breeze. Fonte: [11].

Para uma simulação de valores de potência que podem ser geradas a partir do aerogerador escolhido é necessário definir uma região para obtenção dos dados climáticos. Nesse sentido, foi escolhida a área representada na Figura 4 [12] (área ocupada hoje pela Refinaria do Nordeste RNEST, Petroquímica SUAPE e outros empreendimentos). No entanto, poderia ter sido escolhida qualquer região do mapa, optou-se por essa região porque existem sistemas similares instalados nessa região indicada, ou seja, um exemplo prático da aplicabilidade dos sistemas híbridos e autônomos [13].

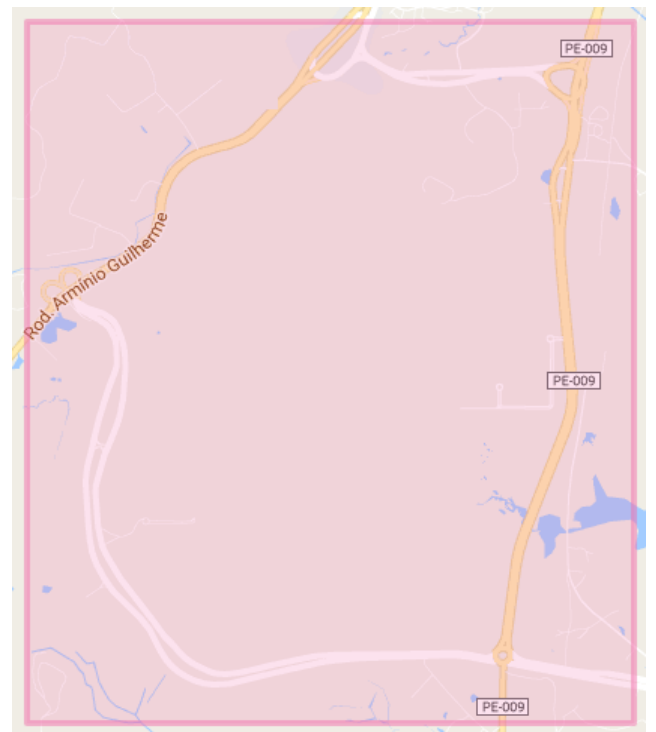

Figura 4: Área escolhida para obtenção de dados climáticos (ponto central aproximadamente $8^{\circ} 22^{\prime} 38.5^{\prime \prime S}$ $35^{\circ} 01^{\prime} 14.2 " W$, ).

Fonte: [12].
Para a obtenção dos dados climáticos foi utilizada a ferramenta on-line SWERA [14]. A partir deste sistema foi construído a Figura 5 e foi obtida a velocidade média anual dos ventos nesta região, cujo o valor foi de $4,78 \mathrm{~m} / \mathrm{s}$.

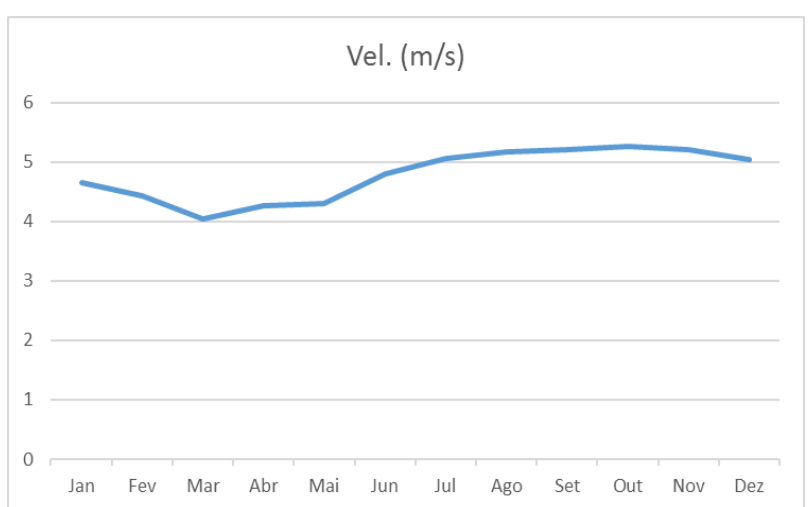

Figura5: Velocidade média mensal para a região da Figura 4.

Fonte: Os autores.

De posse dos dados necessários é possível calcular a potência média estimada que pode ser gerada pelo equipamento:

$$
\begin{gathered}
\mathrm{P}=\frac{1}{2} \mathrm{p}_{x} \cdot A r_{x} V^{3} \\
\mathrm{P}=\frac{1}{2}\left(1_{x} 125\right) \times\left(1_{x} 13\right) \times\left(4 x_{z} 78\right)^{\mathrm{a}}{ }^{\circ}(0,45) \times(0,93)=29_{z} 05 \mathrm{~W} .
\end{gathered}
$$

Neste caso, a energia mensal estimada do aerogerador é de:

$$
\mathrm{E}_{\text {aerogerador }}=29,05 \mathrm{~W} \cdot 24 \mathrm{~h} .30 \text { dias }=20,92 \mathrm{kWh}
$$

Ou seja, considerando o aerogerador escolhido, - sistema fotovoltaico deverá ser capaz de fornecer:

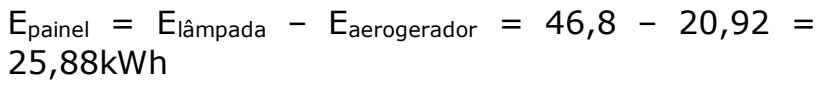
$25,88 \mathrm{kWh}$

Até o momento já foi definida a luminária a ser utilizada como referência e determinado seu consumo estimado de energia elétrica ( $E_{\text {lâmpada }}=$ 46,8 kWh). Também já foi determinada a energia mensal estimada a ser gerada pelo aerogerador escolhido $\quad\left(\mathbb{E}_{\text {aerogerador }}=20,92 \mathrm{kWh}\right)$, e por fim, estimado o valor necessário de energia a ser gerada pelos painéis fotovoltaicos $\left(E_{\text {painel }}=\right.$ $25,88 \mathrm{kWh}$ ). A seguir será detalhado como se calcula o número de painéis solares necessários para suprir a energia a ser gerada. 


\subsection{Energia solar (Painel Fotovoltaico)}

No dimensionamento de um sistema fotovoltaico faz-se necessário a obtenção da radiação solar na localidade onde será instalado o sistema. Para fins de simulação são utilizados os dados da região da Figura 4, obtidos através da ferramenta SWERA [14]. Estes dados estão representados na Figura6.

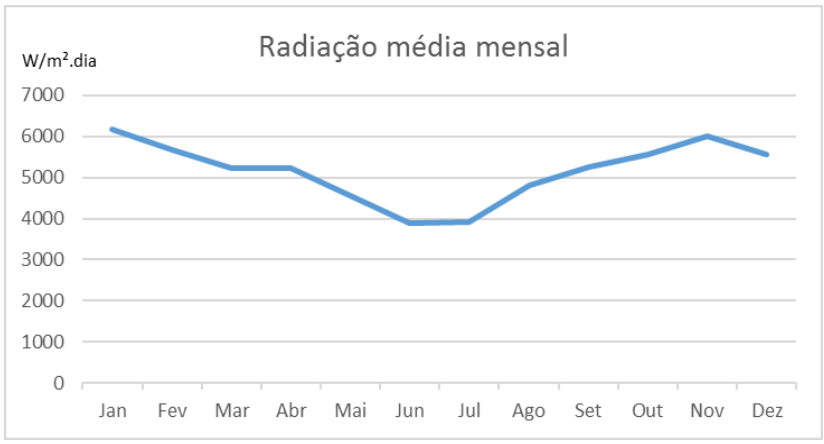

Figura6: Radiação média mensal para a região delimitada na Figura 4.

Fonte: Os autores.

Assim como foi feito para o cálculo da potência média gerada pelo aerogerador será utilizada a radiação média anual, que é $5158 \mathrm{~W} /\left(\mathrm{m}^{2}\right.$.dia). Além dos dados de entrada faz-se necessário escolher um painel fotovoltaico para realizar uma simulação do sistema proposto. Neste caso, o painel escolhido para tal finalidade foi o KD135SXUPU do fabricante KYOCERA [15]. No entanto, poderia ter sido escolhido qualquer outro painel para fins de simulação. Em um estudo aprofundado para a escolha desse equipamento deve-se ponderar as características técnicas e os critérios econômicos para se chegar ao tipo mais adequado para o local de instalação pretendido.

O Quadro 6 apresenta as características desse equipamento:

Quadro 6: Especificação do painel KYOCERA KD135SXUPU.

\begin{tabular}{|c|c|}
\hline Potência & $135 \mathrm{Wp}$ \\
\hline Tensão na máxima potência (Vmp) & $17,7 \mathrm{~V}$ \\
\hline Corrente na máxima potência (Imp) & $7,63 \mathrm{~A}$ \\
\hline Fator de correção do painel fotovoltaico (FC) & 0,834 \\
\hline
\end{tabular}

Fonte: Quadro adaptado de [15] [16].
Considerando que a energia mensal mínima que o painel deverá gerar é $\mathrm{E}_{\text {painel }}=25,88 \mathrm{~kW}$, tem-se [17]:

$$
R_{p V}=V_{m p} I_{m p} S P . F C
$$

Capacidade de geração do painel por dia Sendo o sol pleno [17]:

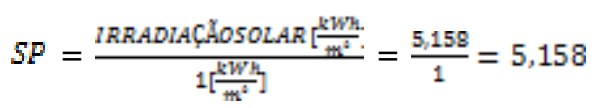

Assim, tem-se:

$P_{P V}=\left(177_{x} 7\right) \cdot(7,63) \cdot(5,158) \cdot(0,834)=580,96 \mathrm{Wh}$

Neste caso, o número de painéis $(\mathrm{N})$ necessários será [17]:

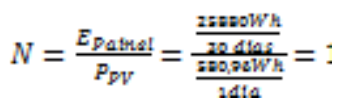

Sendo $1<N<2$, serão necessários 2 painéis do modelo referenciado para o sistema fotovoltaico simulado.

\subsection{Armazenamento de energia (Baterias)}

Além dos sistemas já apresentados, um sistema híbrido e autônomo é composto de um sistema de armazenamento de energia que, neste caso, são as baterias. Estas baterias dão uma confiabilidade nos momentos em que não há vento e nem energia solar.

As baterias possuem uma vida útil que dependem, principalmente, do regime de carga e descarga, além destes fatores, outros parâmetros, como temperatura e idade influenciam diretamente na capacidade e na vida útil do equipamento, o que afeta diretamente o custo de operação e manutenção dos sistemas híbridos e autônomos, pois dos equipamentos envolvidos em tal sistema, a bateria é que possui a menor vida útil. Sendo assim, a bateria pode ser classificada como equipamento mais frágil de um sistema eólico-fotovoltaico autônomo, porque se não for utilizada de forma certa, pode ter sua vida útil drasticamente reduzida [17].

Considerando a facilidade de compra no mercado nacional e o custo reduzido, recomenda- 
se que as baterias de chumbo-ácido seladas sejam utilizadas neste sistema híbridos-autônomo.

$\mathrm{Na}$ escolha do tipo de bateria um dos fatores que, também, deve ser considerado é a profundidade de descarga, que é o valor em porcentagem de energia extraída de uma bateria plenamente carregada em um processo de descarga. Quando esta é máxima diz-se que é a capacidade máxima de corrente que pode ser extraída de uma bateria sem provocar danos [18].

Para a simulação em questão será considerado que o banco de baterias será composto por duas baterias associadas em série para fornecer uma alimentação em $24 \mathrm{Vcc}$ (tensão contínua), que é a mesma tensão definida para a luminária escolhida. Nesse sentido, a capacidade nominal de cada bateria será calculada pela seguinte fórmula adaptada [19]:

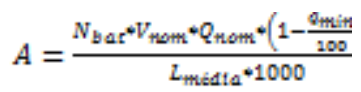

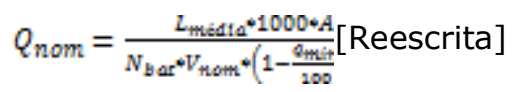

Q: Capacidade nominal de cada bateria (Ah);

: Autonomia do sistema de baterias (h);

I: Número total de baterias do sistema;

v: Tensão nominal de cada bateria (V);

$L_{m}$ : Carga principal média (kWh/dia);

$q_{s}$ estado de carga mínima das baterias (\%).

Com relação a autonomia, de acordo com a resolução normativa n0493 da Agência Nacional de Energia Elétrica (ANEEL), é de, no mínimo, 48 horas para unidades consumidoras atendidas por meio de MIGDI - microssistema isolado de geração e distribuição de energia elétrica ou SIGFI - sistema individual de geração de energia elétrica com fonte intermitente [20]. Nesse sentido, para fins de simulação, será adotada uma autonomia de 48 h.

No que diz respeito ao estado de carga mínimo, os fabricantes recomendam não baixar de uns $30 \%$ a $40 \%$ da capacidade máxima, para que não haja uma descarga excessiva que venha danificar a bateria [18]. Nesse sentido, para fins de simulação será utilizado o valor de $30 \%$. Assim, tem-se:

$$
Q_{\text {nom }}=\frac{1560 * 1000 * 48}{2 \cdot 12 *\left(1-\frac{70}{100}\right) \cdot 24}=\frac{74880}{403,2}=185,7
$$

Considerando um fator de envelhecimento de 1,25 e um fator de correção de temperatura de 0,956 (temperatura inicial de $30^{\circ} \mathrm{C}$ ), tem-se [21]:

Capacidade calculada $=185,71 \times 1,25 \times 0,956$ $=221,92 \mathrm{Ah}$.

Considerando que a capacidade calculadaconsidera uma total ausência de sol e de vento por dois dias, ou seja, qualquer incidência solar ou de vento contribuirá para a carga da bateria. Sendo assim, pode-se considerar que uma bateria de 220Ah atende ao projeto. Neste caso, duas de $220 \mathrm{Ah}$ em série de $12 \mathrm{Vcc}$ ou uma de $220 \mathrm{Ah}$ com tensão de $24 \mathrm{Vcc}$.

\subsection{Controlador de carga}

Os controladores de carga, também conhecidos como reguladores de carga, gerenciador de carga ou regulador de tensão, são dispositivos eletrônicos que apresentam como função básica o controle dos níveis de carga e descarga das baterias. Esse controlador tem a função de desconectar a geração quando a bateria atingir um nível máximo pré-determinado de tensão, e desconectando a carga quando a bateria atingir um nível mínimo de tensão [2].

Em um sistema híbrido, o controlador de carga é um dos principais, se não o principal equipamento do sistema, pois ele é o responsável por gerenciar e controlar o processo de carga e descarga do sistema de baterias, otimizando assim a vida útil delas. Esse equipamento permite que o sistema de baterias seja carregado completamente evitando eventuais sobrecargas e, além disso, monitora a descarga do sistema de baterias para assegurar que não se atinja um nível inseguro [21].

Esse equipamento encarregado de gerenciar o fluxo energético dos geradores para as baterias, e destas para a carga, podem ser de dois tipos: série e paralelo. $O$ do tipo série tem seu funcionamento baseado em interromper a corrente proveniente do aerogerador e dos painéis solares, com o qual cessa o carregamento das baterias. Neste equipamento o dispositivo de controle está em série com geradores e as 
baterias. Já o do tipo paralelo possui um dos dispositivos de controle em paralelo com os geradores, dissipando a energia dos mesmos quando as baterias atingem seu estado de carga máximo [2].

A maior parte dos controladores modernos utilizam-se de duas tecnologias modernas, uma chamada PWM $^{2}$ - Pulse With Modulation ou modulação por pulsos e a outra chamada de MPPT $^{3}$ - Maximum Power Point Tracking. Estas tecnologias são capazes de propiciar a segurança e o melhor aproveitamento das baterias, pois permitem uma fácil reposição da capacidade das baterias $(70 \%$ a $80 \%)$. Porém, o restante $(20 \%$ a $30 \%$ ) requerem circuitos complexos [21].

O controlador de cargadeve possuir a seguinte configuração:

- Entrada para os painéis fotovoltaicos e aerogerador;

- Entrada/Saída para as baterias e saída para a carga (lâmpada);

A Figura 7 mostra como são feitas as conexões entre os equipamentos/dispositivos.

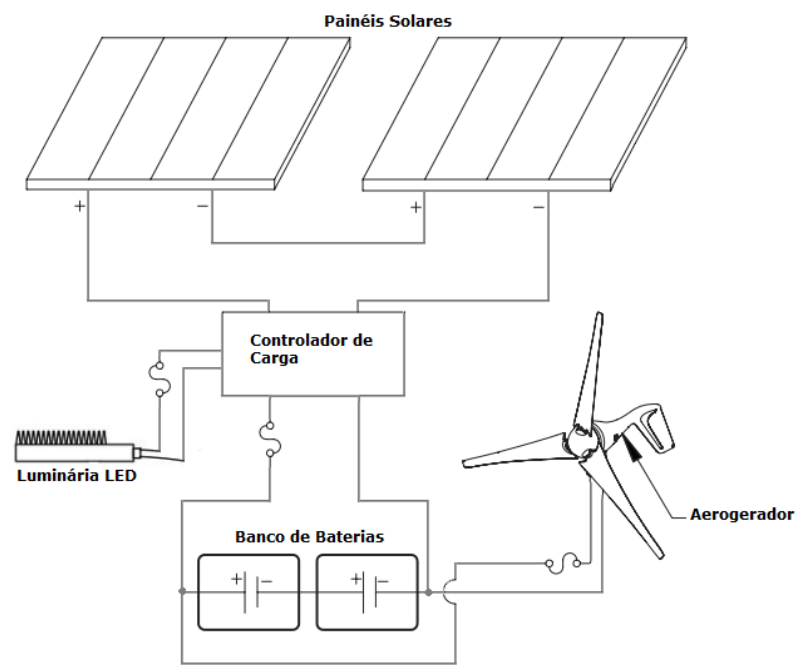

Figura 7: Conexões do controlador de carga. Fonte: Imagem adaptada de [11].

A Figura 7detalha as interligações necessárias

2 PWM ou Pulse WithModulation: Moderna tecnologia de carga que mantém a bateria em sua carga máxima e minimiza a sulfatação da bateria por meio de pulsos de tensão de alta frequência [21].

3 MPPT ou Maximun Power Point Tracker: Método de carga projetado para extrair a máxima energia possível de um módulo solar através da alteração da tensão de operação para maximizar a potência de saída [21]. para o funcionamento adequado, de acordo com o fabricante do aerogerador. Percebe-se pela imagem que o equipamento é interligado diretamente ao sistema de baterias (recomendação expressa apresentada no manual do Air Breeze) [11]. Neste caso, no sistema simulado não há necessidade de um controlador com disponibilidade de interligações para o aerogerador. Assim, o controlador de carga será calculado da seguinte forma [21]:

- corrente na máxima potência para cada painel: 7,63A;

Corrente total $=2 \times 7,63=15,26 \mathrm{~A}$

Na prática, utiliza-se um fator de $25 \%$ para a determinação do controlador a ser utilizado. Neste caso, a corrente final será de 19,075A. Sendo assim, deve-se escolher o controlador de carga que seja superior ao valor encontrado. Comercialmente o mais próximo é o de 20A. Para fins de conclusão do trabalho realizado, é possível adotar o modelo LZP-20-24 (ver Figura 8) da SunLabPower, que é um controlador com saída de alimentação controlada, para uso noturno, com corrente máxima de $20 \mathrm{~A}$ e tensão de trabalho de 24Vcc [23].

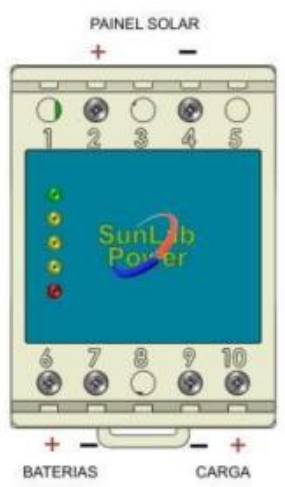

Figura 8: Controlador de carga LZP-20A-24VcC da SunLab Power.

Fonte: SunLab (2015).

\section{CONCLUSÕES}

Esse trabalho apresentou a arquitetura de um sistema híbrido e autônomo de iluminação pública em áreas isoladas ou em áreas que tenham particularidades que exijam soluções não convencionais. Essa arquitetura utiliza fontes renováveis de energia solar e eólica como fontes primárias, baterias como fontes secundárias e a 
fonte luminosa é a base de LEDs. Foram desenvolvidos cálculos simplificados para a determinação dos componentes necessários. Esse sistema apresenta-se como uma alternativa para iluminação de regiões isoladas e de difícil acesso, bem como para trechos de rodovias e trevos, bem como para outras aplicações.

Os cálculos e estudos apresentados neste trabalho foram feitos de forma simplificada e objetiva. Apesar disso, há uma boa proximidade entre os valores encontrados no trabalho em questão e os modelos que estão disponíveis para venda no mercado. No entanto, para a implantação de qualquer sistema de iluminação, seja híbrido ou não, deve-se fazer estudos detalhados para o caso concreto, tanto estudos técnicos específicos, como luminotécnico, quanto de viabilidade financeira.

\section{REFERÊNCIAS}

[1] AGÊNCIA NACIONAL DE ENERGIA ELÉTRICA. Tarifas. Tarifas consumidores. Bandeiras Tarifárias. Disponível em: http://www.aneel.gov.br/bandeirastarifarias. Acesso em: 20 dez. 2016.

[2] HERNÁNDEZ, F. G. Análise experimental e simulação de sistemas híbridos eólicosfotovoltaicos. Tese de Doutorado. Universidade Federal do Rio Grande do Sul, Porto Alegre, 2004. Disponível em: http://www.lume.ufrgs.br/bitstream/handle/ 10183/4569/000412920. pdf? sequence $=1$. Acesso: 18 jun. 2015.

[3] SOLAR-WIND. Hybrid LED Street light. Controller of Solar Wind hybrid street light. Disponível em: http://www.ecosmart.org/productdocs/1-LED-Solar-Wind-Hybrid-Street-Light-Specs-Overview.pdf. Acesso em: 18 jun. 2015.

[4] GREGÓRIO, D.R. Uso de LEDs na iluminação pública. Pós-graduação em iluminação e Design de Interiores. Instituto de Pós-Graduação - IPOG. Especialize. Revista on-line. Janeiro, 2013. Disponível em: https://www.ipog.edu.br/downloadarquivo-site.sp?arquivo =uso-dos-leds-nailuminacao-publica-no-brasil-12153317.pdf. Acesso em: 20 dez. 2016.
[5]CELPE. Grupo Neoenergia. Norma Projeto de Rede de Distribuição de IP com circuito exclusivo. 15 maio 2014.

[6]ASSOCIAÇÃO BRASILEIRA DE NORMAS TÉCNICAS. NBR: 5101: Iluminação pública - Procedimento. Rio de Janeiro: ABNT, 2012.

[7]CONEXLED. Luminária Pública LED. Linha Pequeá (CLP-L30, CLP-L60, CLP-L90 e CLPL120). Disponível em: http://www.conexled.com.br/wpcontent/uploads/2013/02/CLPL30L60L90L120.pdf. Acesso em: 18 jun. 2015.

[8] NEPTUN LIGHT. Product Data Sheets. Solar Lighting Systems. Solar Compatible LED Kometa TM Street Light Fixtures. Disponível em: < http://www.neptunlight.com/catalogs/SOLAR -Catalog-2014.pdf >. Acesso em: $20 \mathrm{dez}$. 2016.

[9] J.E. CHIUSO, A.J. SILVA. Propostas para dimensionamento de um parque eólico na região de Araraquara. Faculdade de Engenharia Mecânica da Universidade Estadual de Campinas, Campinas. Disponível em:

http://www.seeds.usp.br/pir/arquivos/congre ssos/CBPE2004/Artigos/PROPOSTAS\%20PAR A $\% 20$ DIMENSIONAMENTO\%20DE\%20UM\%2 0.pdf. Acesso em: 18jun. 2015.

[10] VERDUM, V. Projeto de Aerogerador com segurança inerente para aplicação urbana. 2013. 107 f. Dissertação (Mestrado em Engenharia Elétrica) Universidade Federal do Rio Grande do Sul, Porto Alegre, 2013. Disponível em: https://www.lume.ufrgs.br/bitstream/handl e/10183/86478/000909136.pdf?sequence $=$ 1. Acesso em: 20 dez. 2016.

[11] PRIMUS WIND POWER, INC. Air Breeze: Manual do Proprietário. Instalação. Funcionamento, Manutenção. Lakewood, Colorado, USA, 2013. Disponível em: http://www.primuswindpower.com/files/13 13/8972/7085/Primus_Air_Manual_Portugu ese.pdf. Acesso em: 15 jul. 2018.

[12] GOOGLE MAPS. Localização central da área onde os dados climáticos foram obtidos. Disponível em:

https://www.google.com.br/maps/place/8 \%C2\%B022'38.5\%22S+35\%C2\%B001'14. 
2\%22W/@-8.3744846,-

$35.0277994,15 \mathrm{z} / \mathrm{data}=! 4 \mathrm{~m} 5 ! 3 \mathrm{~m} 4 ! 1 \mathrm{~s} 0 \times 0: 0 \mathrm{x}$ $0 ! 8 \mathrm{~m} 2 ! 3 \mathrm{~d}-8.3773611 ! 4 d-35.0206111>$.

Acesso em: 20 dez. 2016.

[13] DOIS em um: iluminação do futuro une geração eólica e solar. Plurale em Revista, Ano 5, n. 32, p.59, nov./dez. 2012.

[14] Solar and Wind Energy Resource Assessment (SWERA). Disponível em: http://en.openei.org/apps/SWERA/. Acesso em: 20 dez. 2016.

[15] KYOCERA. Módulo Policristalino de Alta Eficiência. Disponível em:

http://www.kyocerasolar.com.br/site/arqui vos/produtos/68.pdf. Acesso em: $20 \mathrm{dez}$. 2016.

[16] SOUZA, A.C.; MELO, F. C.; MACEDO, J.R.J. Modelagem Computacional de Módulos Fotovoltaicos. PET Elétrica, Universidade Federal de Uberlândia, 2015. Disponível em:

http://www.ceel.eletrica.ufu.br/artigos 2015 /ceel2015_artigo031_r01.pdf. Acesso em: 20 dez. 2016.

[17] SCHUCH, L. et al. Sistema Autônomo de Iluminação Pública de alta eficiência baseado em Energia Solar e LEDs.

Revista Eletrônica de Potência, v. 16, n. 1, p. 17-27, 2011. DOI: http://dx.doi.org/10.18618/REP.2011.1.01 7027. Disponível em:

http://www.academia.edu/14633439/Auto nomous_street_lighting_system_based_on _solar_energy_and_LEDs. Acesso em: $\mathbf{2} 8$ mar. 2015.

[18] SILVA, S. B. Dimensionamento ótimo de sistemas híbridos, com geração fotovoltaica e célula a combustível, para atendimento a comunidades isoladas na Amazônia. 2010. 132 p. Tese (Doutorado em Engenharia Elétrica) Universidade de Brasília, Brasília, DF, 2010. Disponível em:

http://repositorio.unb.br/bitstream/10482/ 8272/1/2010_SergioBatistadaSilva.pdf. Acesso em: 20 dez. 2016.

[19] CRUZ, M. J. Sistemas Híbridos modulares adaptados para redes em expansão. 2010. 140 f. Dissertação (Mestrado Integrado em Engenharia Eletrotécnica e de Computadores Major
Energia ) - Faculdade de Engenharia da Universidade do Porto, 2010. Dissertação realizada no âmbito do Mestrado Integrado, Porto, 2010.Disponível em:https://repositorioaberto.up.pt/bitstream/10216/68181/1/00 0154365.pdf. Acesso em: 20 dez. 2016.

[20] AGÊNCIA NACIONAL DE ENERGIA ELÉTRICA (ANEEL). Resolução Normativa no 493, de 2012. Brasília, jun. 2012. Disponível em:

http://www2.aneel.gov.br/cedoc/ren20124 93.pdf. Acesso em: 20 dez. 2016.

[21] MOURA. Clean Max. Fotovoltaica. Manual e Certificado de Garantia.

[22] HYE WIND POWER ENERGY. Wind solar hybrid street light. Disponível em: http://www.hyenergy.com.cn/a/English/Wi nd_solar_hybrid_street_lighting111. Acesso em: 20 dez. 2016.

[23] LÁBRAMO CENTRONICS IND. E COM. LTDA. DIVISÃO SUNLAB POWER. Manual de Produto. Controlador Solar Inteligente SLC - LZP. Revisão 03/2015. Bragança Paulista, Mar. 2015. Disponível em: http://www.sunlab.com.br/manuais/MANU AL\%20DE\%20INSTALA\%C3\%87\%C3\%830 \%20\%20-\%20SLC_LZP\%20rev3_2015.pdf. Acesso em: 20 dez. 2016. 